\title{
ADRENAL TUMORS AND CUSHING'S SYNDROME - OUR SURGICAL EXPIRIENCE
}

\author{
Ignatov V., K. Ivanov, N. Kolev, A. Tonev \\ Department of General and Operative Surgery, University Hospital "St. Marina"
}

\author{
Reviewed by: Assoc. Prof. Kr. Hristozov
}

\begin{abstract}
Cushing's syndrome is a clinical condition of excess cortisol production secondary to disorders of the pituitary, adrenal glands, or ectopic production of adrenocorticotrophic hormone (ACTH). The syndrome is manifested by obesity, hypertension, diabetes, amenorrhea, and other symptoms and without recognition and proper treatment can result in substantial morbidity and mortality. For patients who do not respond to treatment directed at pituitary adenomas, have occult ectopic ACTH secreting tumors, or have primary bilateral adrenal disorders such as primary pigmented nodular adrenal disease or macronodular adrenal hyperplasia, bilateral adrenalectomy is effective in curing or controlling hypercortisolism and reversing the signs and symptoms of excess cortisol production $(1,2)$. The morbidity associated with bilateral adrenalectomy is not inconsequential, and reported mortality rates range from $2 \%$ to $5 \%$ (3-6). In general, patients with Cushing's syndrome are predisposed to infectious or hemorraghic complications as well as to impaired wound healing. The open operative approache, used in our clinic for adrenalectomy is the transabdominal approach through either a midline or bilateral subcostal incision (7). The anterior approach allows a complete abdominal and retroperitoneal exploration and may be most appropriate in patients in whom a coincidental abdominal procedure is contemplated $(9,10)$. In particular, because many of these patients are relatively young at the time of treatment and return to fully productive lives after surgery.
\end{abstract}

\section{MATERIAL}

We present an evaluation of 18 patients, operated in Clinic of general and operative surgery in the period of 1999-2008. All of them had tumors of the suprarenal glands.

\section{DIAGNOSIS}

Biochemical diagnosis of Cushing's syndrome was confirmed by serum and urinary cortisol levels in conjunction with serum ACTH. In patients who had not responded to treatment for pituitary lesions or who had ectopic ACTH production, serum ACTH was equivalent. Adrenal disease was confirmed radiographically by a combination of computed tomographic scans, magnetic resonance imaging scans (Fig.1, Fig.2, Fig.3).

\section{Operative Treatment}

All operations were performed by a single operating team. The incision for the anterior approach was either a midline $(\mathrm{n}=16)$ or bilateral subcostal $(\mathrm{n}=11)$ incision depending on body habitus. With the anterior approach, a Kocher maneuver and mobilization of the right lobe of the liver was used to gain access to the right adrenal gland. Left adrenal

Address for correspondence:

Kr.Ivanov, Varna, $1 \mathrm{Hr}$. Smirnenski str,

University Hospital "St. Marina"

e-mail: teraton@abv.bg, kivanov@gisurgery.bg gland typically was approached through the lesser sac and exposed by reflecting the pancreas superiorly. A standard regimen of tapered perioperative stress dose steroids was administered in all patients followed by maintenance hydrocortisone orally. With excising significantly larger adre-

\begin{tabular}{|l|c|}
\hline \hline Type of Complication & $\mathbf{n}(\%)$ \\
\hline Incidental splenectomy & $1(5)$ \\
\hline Upper pole renal infarction & $1(5)$ \\
\hline Reoperation (bleeding) & $1(5)$ \\
\hline Vascular injury & $2(10)$ \\
\hline Wound infection & $3(15)$ \\
\hline Chronic back pain & $2(10)$ \\
\hline Incisional numbness & $3(15)$ \\
\hline Chronic fatigue & $2(10)$ \\
\hline Incisional hernia & $2(10)$ \\
\hline \hline
\end{tabular}

nal glands via the anterior approach, the operative blood loss was identical with a trend toward a lower transfusion requirement with anterior incisions. After surgery, the patients had either no nasogastric suction or at most 1 day af- 
ter anesthesia, whereas gastric decompression was continued for a mean of 2.1 days. Patients proceed to independent walking $>50 \mathrm{ft}$ routinely by the second postoperative day. The average daily analgesic doses per patient on the day of surgery and the first 2 postoperative days were as the other operative procedures in the abdomen and didn't advocate increase. Oral analgesia was used by a majority of patients in both groups by the third postoperative day. The intraoperative and early postoperative complications were as follow:

The operated group incurred two operative complications, including splenic injury requiring splenectomy and a reoperation for postoperative bleeding from the right adrenal bed in a patient with an unappreciated coagulopathy. Superficial wound infections developed in two patients, who were treated by having the wound opened, dressing changes, and antibiotics for healing by secondary intention. Postoperative mechanical ventilation was required in one patient after a posterior adrenalectomy and in three patients in the anterior group, primarily due to severe Cushing's syndrome with muscle weakness. Late complications differ with the greatest point of distinction being chronic back pain. Only $7 \%$ of the group reported mild back pain. This late complication translated into a significantly prolonged time to return to work after the operative procedure. Another late complication was a high rate of incisional hernia of $10 \%$. Other complications that were fairly minor but noted were incisional numbness and chronic fatigue.

\section{DISCUSSION}

In this study, we reviewed the intraoperative and shortterm and long-term postoperative outcomes of adrenalectomy through an anterior approach for Cushing's syndrome. Patients in our group were evaluated by weight, pathologic diagnoses, and severity of hypercortisolism (serum cortisol, urinary cortisol). Despite the technical difficulties in removing larger glands in patients undergoing adrenalectomy, there was no significant major differences with some authors (1-9) with respect to operative time, estimated blood loss, or transfusion requirements. The timing of conversion to oral analgesics was not significantly different according to other groups of patients. The slightly increased time to begin clear liquids and advance to a regular diet reflects a longer ileus after a transabdominal procedure. Length of hospitalization was not considered an appropriate endpoint of comparison as many patients remain hospitalized at our institution after surgery for a brief period for research purposes or because they have considerable distances to travel home. The intraoperative complications and the acute postoperative morbidity were similar with the literature review (1-9). The vascular injuries in the our group occurred in obese patients undergoing explorations via relatively small incisions but caused no secondary problems for these patients. The reported wound infection rate after adrenalectomy ranges from $4 \%$ to $23 \%$ (7), and the overall infection rate of $12 \%$ ( 6 of 48 patients) in this series is not inconsistent with previous results. Late morbidity was evaluate. All patients were contacted by telephone and asked to describe any chronic symptoms relating to the procedure. The responses from a similar telephone interview of the patients who had undergone anterior operations were distinctly different. Therefore, the difference in back pain cannot be attributed to osteoporosis or muscle injury due to the cortisol excess of Cushing's syndrome, but rather is a direct result of the paraspinous incisions used for the posterior approach. The only significant late complication that could be emphasized was incisional hernia in 2 of 18 patients. This high rate of hernia (11\%) may be explained by poor wound healing due to cortisol excess and the obesity associated with this disease. During the past few years, there has been considerable interest in the use of laparoscopic adrenalectomy (12-14). However, the utility of laparoscopic bilateral adrenalectomy has not yet been clearly shown, particularly in patients with Cushing's syndrome who almost uniformly have significant truncal obesity and significant retroperi- toneal fat. Several distinct potential drawbacks of this procedure may be the technical difficulties in removing large lesions, the longer operative time, and the carbon dioxide intoxication (5). The use of the laparoscopic approach has not been advocated for patients with tumors greater than $70 \mathrm{~g}$ or those with central obesity (3). Some investigators think that the posterior approach to adrenalectomy allows recovery nearly equivalent to that expected with bilateral laparoscopic adrenalectomy. Our data support this impression, may be intuitively expected, particularly with the widespread use of epidural catheters for postoperative pain control after laparotomy. The impression of a fast recovery with less pain using the posterior approach is overshadowed by the very significant incidence of chronic pain reported in our series. Unless the operating surgeon continues to observe these patients who are typically managed long term by an endocrinologist, this late complication may not be appreciated. We conclude that adrenalectomy is a safe techniques for the treatment of Cushing's syndrome. However, many patients are relatively young at the time of treatment (the mean age in this series was younger than 40 years of age) and will return to full and productive lives after surgery. The data presented here show that the majority of patients who undergo posterior bilateral adrenalectomy will have long-term incisional pain, which can be severe and incapacitating in some.

\section{REFFERENCES}

1. Zeiger MA, Fraker DL, Pass HI, et al. Effective reversibility of the signs and symptoms of hypercortisolism by bilateral adrenalectomy. Surgery 1993; 114:1138-1143.

2. Sarkar R, Thompson NW, McLeod MK. The role of adrenalectomy in Cushing's syndrome. Surgery 1990; 108:1079-1084.

3. Welboum RB, Manolas KJ. The role of adrenalectomy in the management of Cushing's syn- 
drome. In: Johnston L, ed. Endocrine Surgery. Kent: Butterworth \& Co; 1983:53-75.

4. Cohen KL, North RH, Pechinski T. Incidence of pituitary tumors following adrenalectomy: a long-term follow-up study of patients treated for Cushing's disease. Arch Intern Med 1978; 138:575-579.

5. O'Riordain DS, Farley DR, Young WF, Jr, et al. Long-term outcome of bilateral adrenalectomy in patients with Cushing's syndrome. Surgery 1994; 116:1088-1094.

6. Lairmore TC, Ball DW, Baylin SB, Wells SA. Management of pheochromocytomas in patients with multiple endocrine neoplasia type 2 syndromes. Ann Surg 1993; 217:595-603.

7. van Heerden JA, Young WF, Jr, Grant CS, Carpenter PC. Adrenal surgery for hypercortisolism-surgical aspects. Surgery 1995; 117:466-472.

8. van Heerden JA, Sheps SG, Hamberger B, et al. Pheochromocytoma:Current status and changing trends. Surgery 1982; 91:367-373.
9. Russell CF, Hamberger B, van Heerden JA, et al. Adrenalectomy:anterior or posterior approach? Am J Surg 1982; 144:322-324.

10. Edis AJ, Ayala LA, Egdahl RH. Manual of endocrine surgery. NewYork: Springer-Verlag, 1975.

11. Cheatham ML, Chapman WC, Key SP, Sawyers JL. A meta-analysis of selective versus routine nasogastric decompression after elective laparotomy. Ann Surg 1995; 221:469-478.

12. Suzuki K, Kageyama S, Ueda D, et al. Laparoscopic adrenalectomy:Clinical experience with 12 cases. J Urol 1993; 150:1099-1102.

13. Gagner M, Lacroix MD, Prinz RA, et al. Early experience with laparoscopic approach for adrenalectomy. Surgery 1993; 114:1120-1125.

14. Go H, Takeda M, Takahashi H, et al. Laparoscopic adrenalectomy for primary aldosteronism: a new operative method. J Laparoendosc Surg 1993; 3:455-459.

15. Evans RM, Hulbert JC, Reddy PK. Complications of laparoscopy. Semin Urol 1992; 10:164-168. 